\title{
Finite Density Simulation with Canonical Ensemble
}

\section{$\chi$ QCD Collaboration}

\section{Anyi $\mathbf{L i}^{*}$}

Department of Physics and Astronomy, University of Kentucky, Lexington KY 40506, USA

E-mail: anyiliepa.uky.edu

\section{Xiangfei Meng}

Department of Physics and Astronomy, University of Kentucky, Lexington KY 40506, USA

Department of Physics, Nankai University, Tianjin 300071, China

Email: mengxf@mail.nankai.edu.cn

\section{Andrei Alexandru}

Physics Department, The George Washington University, Washington, DC 20052, USA

E-mail: aalexan@gwu.edu

\section{Keh-Fei Liu}

Department of Physics and Astronomy, University of Kentucky, Lexington KY 40506, USA

E-mail: liulpa.uky.edu

\begin{abstract}
QCD at non-zero baryon density is expected to have a critical point where the zero-density crossover turns into a first order phase transition. To identify this point we scan the density-temperature space using a canonical ensemble method. For a given temperature, we plot the chemical potential as a function of density looking for an "S-shape" as a signal for a first order transition. We carried out simulations using Wilson fermions with $m_{\pi} \approx 1 \mathrm{GeV}$ on $6^{3} \times 4$ lattices. As a benchmark, we ran four flavors simulations where we observe a clear signal. In the two flavors case we do not see any signal for temperatures as low as $0.83 T_{c}$. Preliminary results for the three flavor case are also presented.
\end{abstract}

The XXVI International Symposium on Lattice Field Theory

July 14-19 2008

Williamsburg, Virginia, USA

\footnotetext{
${ }^{*}$ Speaker.
} 


\section{Introduction}

In recent years, full QCD simulations have become feasible due to development of new algorithms and increasing computational power. Lattice simulations using dynamical fermions can now be performed at finite temperature and zero baryon density. However, simulations at non-zero baryon density remain a challenge for lattice QCD due to the complex nature of the fermionic determinant where the conventional Monte Carlo methods fail. The standard solution of splitting the action into the real and positive part and an extra phase fails due to sign and overlap problems. To address the overlap problem, a method based on the canonical partition function has been proposed [1]. While expensive - every update involves the evaluation of the fermionic determinant - finite baryon density simulations based on this method proved feasible [2] and a program was outlined to scan the QCD phase diagram to look for the critical point $[3,4]$.

In this paper, we present results based on simulations on $6^{3} \times 4$ lattices with Wilson fermions. We plot the chemical potential as a function of baryon density and we clearly observe the "Sshape" structure in the $N_{f}=4$ case, indicating a first order phase transition [8]. We do not see such a structure in the $N_{f}=2$ case down to $0.83 T_{c}$. We will also present preliminary results for $N_{f}=3$.

\section{Algorithm}

The simplest way to show how to build the canonical ensemble in Lattice QCD is to start from the fugacity expansion,

$$
Z(V, T, \mu)=\sum_{k} Z_{C}(V, T, n) e^{\mu k / T},
$$

where $k$ is the net number of quarks (number of quarks minus the number of anti-quarks) and $Z_{C}$ is the canonical partition function. Using the fugacity expansion, it is easy to see that we can write the canonical partition function as a Fourier transform of the grand canonical partition function,

$$
Z_{C}(V, T, k)=\left.\frac{1}{2 \pi} \int_{0}^{2 \pi} \mathrm{d} \phi e^{-i k \phi} Z(V, T, \mu)\right|_{\mu=i \phi T} .
$$

As an illustration, we will consider the case of two degenerate flavors. After integrating out the fermionic part, we get a simple expression

$$
Z_{C}(V, T, k)=\int \mathscr{D} \mathrm{U} e^{-S_{g}(U)} \operatorname{det}_{k} M^{2}(U),
$$

where

$$
\left.\operatorname{det}_{k} M^{2}(U) \equiv \frac{1}{2 \pi} \int_{0}^{2 \pi} \mathrm{d} \phi e^{-i k \phi} \operatorname{det} M(m, \mu ; U)^{2}\right|_{\mu=i \phi T},
$$

is the projected determinant with the fixed net quark number $k \cdot \operatorname{det}_{k} M^{2}(U)$ is a real number due to the charge conjugation symmetry of the canonical partition function. However, it is not necessarily positive. In our simulations, we use $\left|\operatorname{Re}_{\operatorname{det}_{k}} M^{2}(U)\right|$ and fold the phase factor in the observables.

Exact determinant calculation of fermion matrix is very demanding even on $6^{3} \times 4$ lattices. An alternative is to use a noisy estimator $[5,7]$ but this is quite cumbersome. For simplicity sake, we used exact evaluation of the determinant in this study. Another technical problem has to do with 
the Fourier transform; our original approach was to use an approximation where we replaced the continuous definition with a discrete one, i.e.:

$$
\operatorname{det}_{k} M^{2}(U) \approx \frac{1}{N} \sum_{j=0}^{N-1} e^{-i k \phi_{j}} \operatorname{det} M\left(U_{\phi_{j}}\right)^{2}, \quad \phi_{j}=\frac{2 \pi j}{N} .
$$

It was shown that the errors introduced by this approximation are small [4] at least for small quark numbers. There are two problems with this approach: the computation time increases linearly with the net quark number and, for large enough densities, the Fourier components become too small to be evaluated using double precision floating point numbers. To address these issues, in this study we used a different approximation method: the winding number expansion [6]. This method is both faster and more accurate than the original method.

As mentioned above, we used the chemical potential to probe the phase transition. In the canonical approach the chemical potential is measured as the increase in free energy when we introduce one more baryon in the system, i.e.:

$$
\langle\mu\rangle_{n_{B}}=\frac{F\left(n_{B}+1\right)-F\left(n_{B}\right)}{\left(n_{B}+1\right)-n_{B}}=-\frac{1}{\beta} \ln \frac{Z_{C}\left(3 n_{B}+3\right)}{Z_{C}\left(3 n_{B}\right)}=-\frac{1}{\beta} \ln \frac{\langle\gamma(U)\rangle_{o}}{\langle\alpha(U)\rangle_{o}}
$$

where

$$
\begin{aligned}
\alpha(U) & =\frac{\operatorname{Redet}_{3 n_{B}} M^{2}(U)}{\left|\operatorname{Redet}_{3 n_{B}} M^{2}(U)\right|} \text { and } \\
\gamma(U) & =\frac{\operatorname{Re} \operatorname{det}_{3 n_{B}+3} M^{2}(U)}{\left|\operatorname{Redet} \operatorname{det}_{3 n_{B}} M^{2}(U)\right|} .
\end{aligned}
$$

$\alpha(U)$ is the phase and \langle\rangle$_{o}$ stands for the average over the ensemble generated with measure $\left|\operatorname{Redet}_{3 n_{b}} M^{2}(U)\right|$. If the configurations in the ensemble have equal probability for both positive and negative $\alpha(U)$, the denominator in the equation above becomes zero and we have a "sign problem". In our simulations the sign oscillations are under control.

\section{QCD phase diagram}

At zero baryon density, it has been known for quite some time that QCD undergoes a transition from a confined phase to a deconfined phase at a temperature $T_{c} \approx 170 \mathrm{MeV}$. Lattice QCD suggests that the transition is in fact a smooth crossover. This is expected to turn into a first order phase transition as the baryon density is increased. A schematic picture of the expected phase diagram in the density-temperature plane (see Fig. 1) shows the crossover ending with a critical point at some non-zero baryon density. The first order phase transition is characterized by a coexistence region separating the hadronic phase and the plasma phase.

To search for the phase boundaries of the coexistence region, we scan the phase diagram by varying the density while keeping the temperature fixed. The baryon chemical potential should exhibit an "S-shape" as one crosses the coexistence region [8].

In our simulations we use quarks that are much heavier than the physical quarks. Furthermore, for simplicity sake, we carry out simulations where the quark masses are degenerate. We will

present results for simulations using 2, 3 and 4 degenerate flavors of quarks. The expected phase 


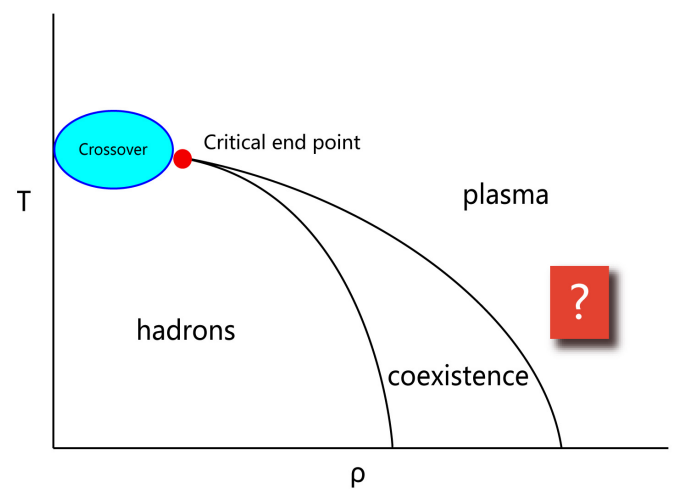

Figure 1: Schematic phase diagram of QCD.
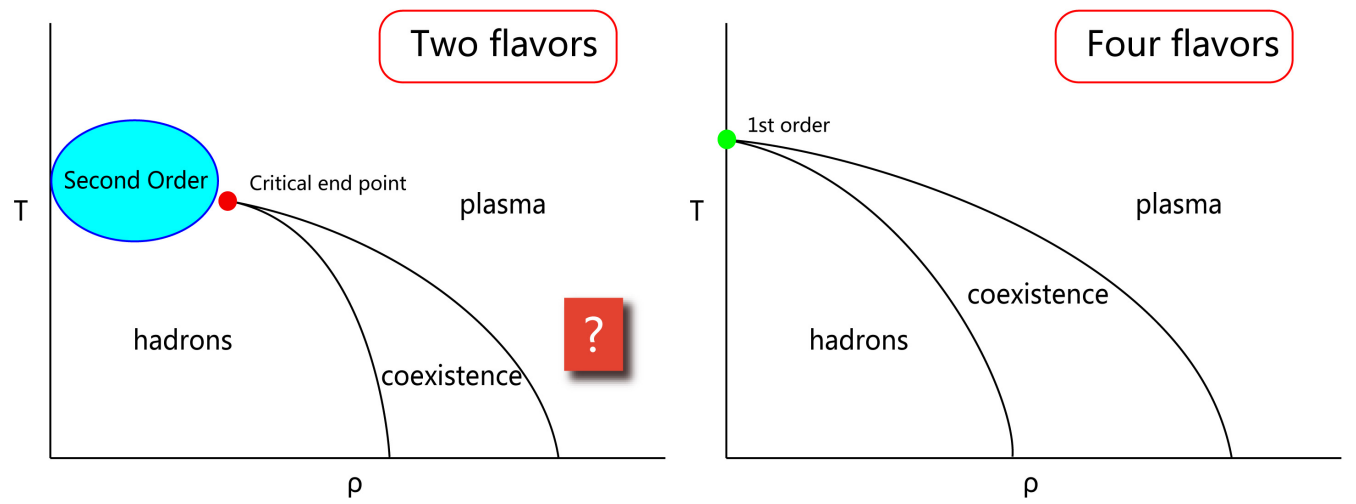

Figure 2: Expected phase diagrams of two flavors (left) and four flavors (right).

diagrams for 2 and 4 flavor cases are shown in Fig. 2. The two flavor case is expected to have a diagram very similar to the full QCD one whereas it is known that in the case of four flavors the first order phase transition extends all the way to zero baryon density. We will use the four flavor simulations as a benchmark to show that the methodology we use can determine the boundaries of the coexistence region.

\section{Results}

In Fig. 3 we show the results for our four flavor simulation. On the technical side, we note that we didn't have a sign problem: even the simulations at the largest density where the box size is $1.8 \mathrm{fm}$ and the temperature is $0.90 T_{c}$, the sign oscillations were moderate. The plots show a clear signal for a first order phase transition when the temperatures are lower than $T_{c}$.

To identify the boundaries of the coexistence region and the critical value for the chemical potential we used the Maxwell construction [8]. More precisely, we selected four points in the "S-shape" region and we fit these points with a third order polynomial. A better approach would be to use some phenomenologically motivated functional form and try to fit a larger region; we changed the fit function and we also extended the fit region. For all reasonable fits, we found that the values of the boundary points, $\rho_{1}$ and $\rho_{2}$, and the value of the critical chemical potential, $\mu_{c}$, are 


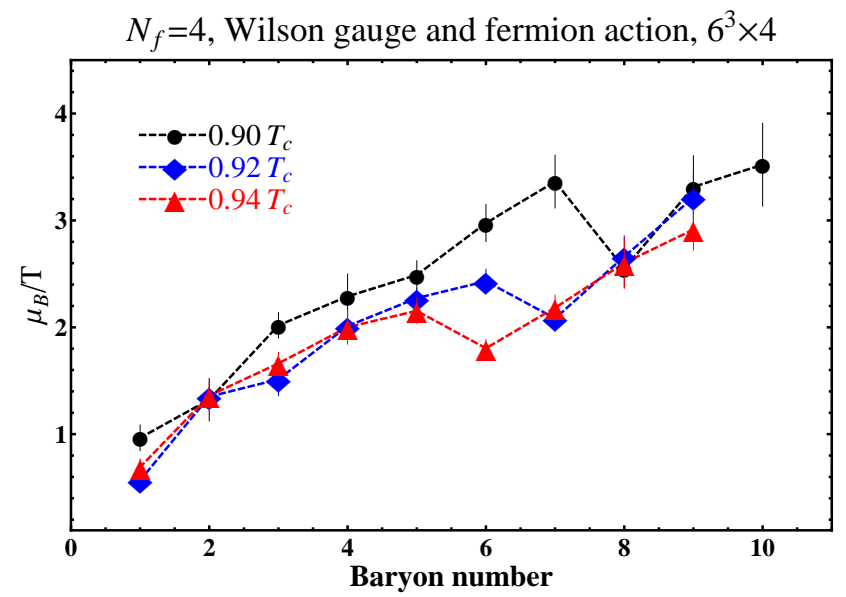

Figure 3: Baryon chemical potential vs. baryon number at different temperatures for $N_{f}=4$.

fairly insensitive to our choice of the fit function or fit region - the simple third order polynomial fit was sufficient.

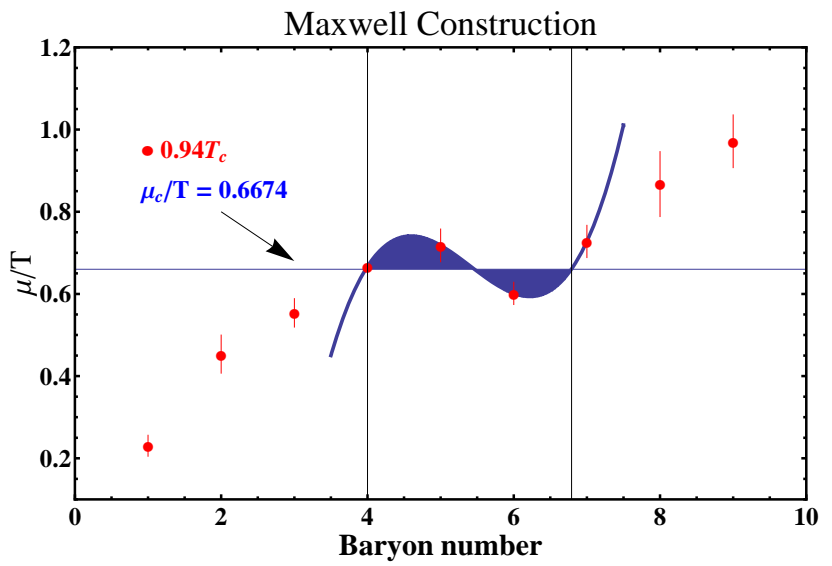

Figure 4: Maxwell construction for $T=0.94 T_{c}$ and $N_{f}=4$.

Once the $\rho_{1}$ and $\rho_{2}$ are determined for different temperatures, they can be used to plot the boundaries of the coexistence region. We can also find the critical point by determining where the width of the coexistence region shrinks to zero. In Fig. 5 we compare our results to those from a study using staggered fermions [8]. We see that our coexistence region is much narrower. This could be due to our heavier pion mass ( $m_{\pi} \approx 1 \mathrm{GeV}$ compare to $m_{\pi} \approx 300 \mathrm{MeV}$ ), or due to the fact that we use a different fermion formulation.

The fact that the results of our four flavors simulations are consistent with the expectation and with other lattice studies is encouraging. The only issue that needs to be addressed is the discrepancy in the location of the boundaries.

In Fig. 6 we show our results for $N_{f}=2$ simulations. These simulations are more expensive than the four flavors simulations due to sign fluctuations. In the two simulations that we ran at $T=0.86 T_{c}$ and at $T=0.83 T_{c}$ we do not see any signal for a first order phase transition. There is at 


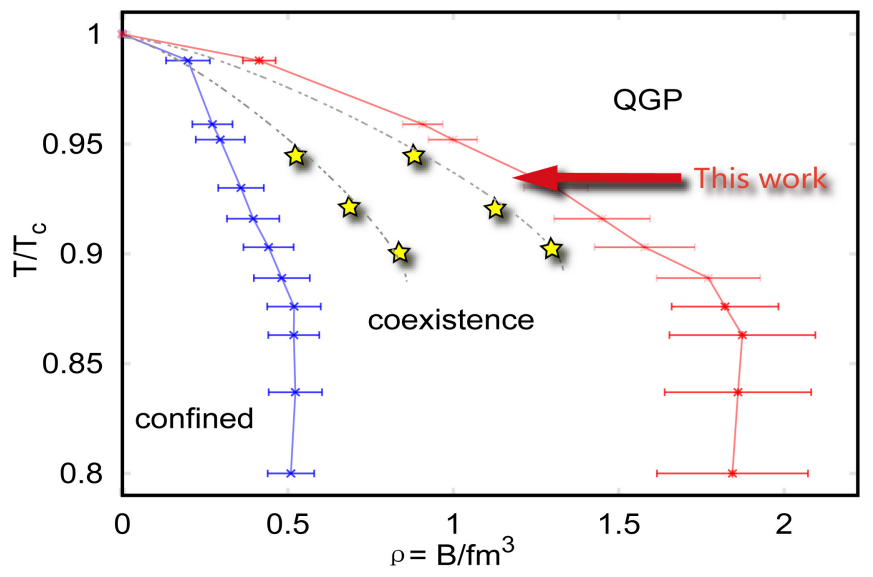

Figure 5: Phase boundary for four flavors QCD: our work and the results of a study using staggered fermions [8]. (Dashed lines are plotted to guide the eye; they are not the result of an extrapolation. The error bars are not determined yet.)

least one claim [9] that the critical point occurs at temperatures as low as $T=0.8 T_{c}$. If this is indeed the case, we need to run simulations at even lower temperatures in order to see the "S-shape".

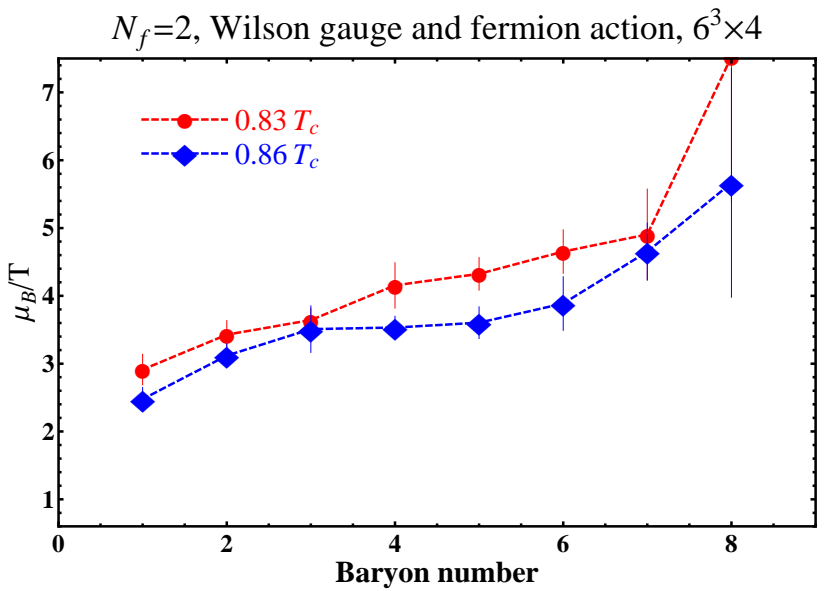

Figure 6: Baryon chemical potential vs. baryon number for $N_{f}=2$.

Three quark flavors are relevant for the structure of matter at energies of the order of few hundred MeV: two light quarks $u, d$ and one heavier quark $s$. Lattice simulations that come close to approximate full QCD treat the lighter quark flavors as degenerate and use one heavier quark flavor. Ideally, we would like to carry out simulations close to the physical point. However, this is not really practical especially for Wilson fermions on lattices as coarse as we use in our study. In fact, our quark masses are even heavier than the strange quark mass. We decided to investigate the $N_{f}=3$ with the hope that the phase diagram is, at least qualitatively, close to the full QCD phase diagram.

In Fig. 7 we show the results of our simulations for $N_{f}=3$. For these simulations we used Iwasaki gauge action and clover fermions in order to reduce lattice discretization errors. For $T=$ $0.92 T_{c}$, we don't see any signal for a first order phase transition. 


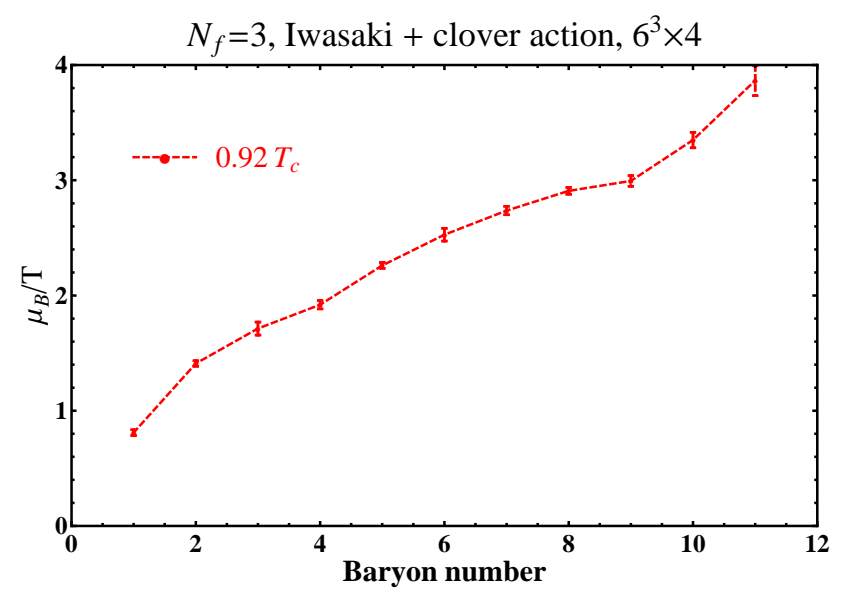

Figure 7: Baryon chemical potential vs. baryon number at different temperatures for four flavors. Simulations are done using Iwasaki gauge action and clover fermion action.

\section{Summary}

We presented results for QCD simulations at non-zero baryon density using the canonical ensemble approach. In the four flavor case we see a clear signal for a first order phase transition this is consistent with phenomenological expectations and previous lattice results. This proves that our method is sound. In the two flavor case, we do not see any signal for temperatures as low as $0.83 T_{c}$. We also presented results for three flavors where we do not find any signal for a transition at $T=0.92 T_{c}$. We plan to continue our investigations at lower temperatures and smaller quark masses.

\section{References}

[1] K. F. Liu, Edinburgh 2003, QCD and Numerical Analysis Vol. III (Springer, New York, 2005) 101 arXiv:hep-lat/0312027.

[2] A. Alexandru, M. Faber, I. Horváth, K. F. Liu, Phys. Rev. D72 (2005) 114513 arXiv:hep-lat/0507020.

[3] A. Li, A. Alexandru, K. F. Liu, PoS (LATtICE 2006) 030 arXiv:hep-lat/0612011.

[4] A. Li, A. Alexandru, K. F. Liu, PoS (LATtICE 2007) 203 arXiv:0711.2692 [hep-lat].

[5] A. Alexandru, A. Li, K. F. Liu, Pos (LAttiCE 2007)167 arXiv:0711.2678 [hep-lat].

[6] X. Meng, A. Li, A. Alexandru, K. F. Liu, PoS (LATtiCE 2008) 032.

[7] B. Joo, I. Horváth, and K. F. Liu, Phys. Rev. D67 (2003) 074505.

[8] Ph. de Forcrand, S. Kratochvila, Nucl. Phys. B (Proc. Suppl.) 153 (2006) 62.

[9] S. Ejiri, arXiv:0804.3227 [hep-lat]. 